\title{
A Proposed Grading System and Experience of COVID-19 Associated Rhino Orbito Cerebral Mucormycosis from an Indian Tertiary Care Center
}

\author{
Neelam Vaid ${ }^{1}$ Prasun Mishra ${ }^{2}$ Nikhil Gokhale ${ }^{1} \cdot$ Sanjay Vaid $^{3}$. \\ Varada Vaze $^{1} \cdot$ Ajay Kothadiya $^{1} \cdot$ Trisha Deka $^{1} \cdot$ Rashmi Agarwal $^{1}$
}

Received: 25 October 2021/Accepted: 8 November 2021/Published online: 15 November 2021

(C) Association of Otolaryngologists of India 2021

\begin{abstract}
Mucormycosis is an opportunistic fungal infection. India faced an unprecedented increase in patients with post coronavirus disease 2019 (COVID-19) associated rhino-orbito-cerebral mucormycosis (ROCM). This study proposes a grading system which correlates the extent of the disease with the management plan. An observational study was conducted January 2021-June 2021. We identified 65 patients. Eleven patients had mild disease, 27 patients had moderate, 16 patients were severe and 11 patients were graded as very severe. The management was planned based on this grading system. Early diagnosis,
\end{abstract}

Prasun Mishra

entprasun@gmail.com

Neelam Vaid

neelu1964@gmail.com

Nikhil Gokhale

nikhilgokhale@yahoo.com

Sanjay Vaid

svaidhn@gmail.com

Varada Vaze

varadavaze@gmail.com

Ajay Kothadiya

drajay1971@gmail.com

Trisha Deka

drtrishadeka@gmail.com

Rashmi Agarwal

drrashmi65@gmail.com

1 Department of Otorhinolaryngology, KEM Hospital, Pune, Maharashtra, India

2 Department of Otorhinolaryngology, Bharati Vidyapeeth Medical College, Pune, Maharashtra, India

3 Head Neck \& ENT Division, Star Imaging and Research Centre, Pune, Maharashtra, India aggressive surgical debridement and antifungal drug therapy is the key to improve survival in ROCM. Procedures such as endoscopic orbital clearance, sublabial maxillectomy, and modified endoscopic Denkers (MED) approach facilitate access and surgical debridement. The new grading system proposed assists in planning the approach and extent of surgical debridement.

Keywords COVID-19 · Mucormycosis · Grading

\section{Introduction}

Mucormycosis is an opportunistic fungal infection caused by group of filamentous molds from the order Mucorales within the zygomycete family. They are involved in infections of different body parts, such as the nose and sinuses, eyes, brain, lungs, gastrointestinal tract and skin. They mainly affect individuals with underlying immunocompromised conditions. Only $6-10 \%$ of cases occur in subjects without underlying disease. The pathological feature of this infection is angio-invasion, thrombosis and subsequent necrosis of tissue. The treatment of mucormycosis is control of the immunosuppressive state, early and aggressive surgical debridement and antifungal therapy [1-3]. Chamilos et al. have reported a mortality rate of $46 \%$ in patients with mucormycosis [4].

The incidence of mucormycosis in the literature world wide varies from 0.005 to 1.7 per million population [5]. Epidemiological studies have reported the prevalence in India to be 80 times higher in the preCOVID era ( 0.14 per 1000) $[6,7]$.

An alarming increase in cases with ROCM and COVID 19 infection was seen especially during the second wave COVID 19 pandemic in India. Numerous risk factors such 
as uncontrolled diabetes mellitus, immunocompromised states (due to administration of corticosteroids) and iron overload were considered responsible for this notifiable epidemic of COVID 19 associated ROCM.

The management of such a large number of mucormycosis patients was a challenge to an already stretched medical infrastructure associated with a scarcity of injection amphotericin. We devised a grading system based on clinical evaluation and imaging to optimize treatment protocols. This enabled a standardized level of care to all the patients.

\section{Methods}

This was an observational study performed at a tertiary care teaching hospital from January 2021-June2021. All patients who presented with a clinical suspicion of ROCM were evaluated in the ENT OPD. An expedited institutional ethical committee clearance was obtained for the study. A written informed consent was taken from all patients admitted for ROCM for being part of the study.

\section{ENT OPD Protocol}

A thorough history was taken, with specific focus on history of facial pain, dental pain and loose teeth, loss of sensation of the cheek, blurred vision, diplopia, blindness, and headache.

Diagnostic nasal endoscopy was performed for all patients to look for the presence of black eschar or crusts.

Nasal swabs from the middle turbinate were obtained and sent for $10 \%$ potassium oxide $(\mathrm{KOH})$ staining.

All patients were referred for imaging. Contrast enhanced computerized tomography (CT) scan was used as the primary imaging modality. Patients with suspected intraorbital, neurovascular or intracranial involvement were referred directly for contrast enhanced Magnetic resonance imaging (MRI).

The CT scan protocol is outlined in Table 1 and the MRI protocol is outlined in Table 2.

A team consisting of ENT surgeons, physicians, ophthalmologists, pathologists, microbiologists and, infectious disease specialists reviewed the reports of all patients with confirmed RCOM. A management plan for each patient was formulated based on the grading of the disease. Table 3 gives the grading system used and the proposed treatment options.

\section{Treatment}

On admission, patients and their families were counselled regarding the nature of the disease and the need for a combined modality of treatment comprising of medication and surgical debridement.

In all patients suspected to be suffering from ROCM intravenous (IV) amphotericin was started immediately. IV liposomal amphotericin was started at a dose of $5-10 \mathrm{mg} /$ $\mathrm{kg}$ and continued till patients showed signs of recovery clinically and radiologically. Due to the sudden surge in cases and nationwide shortage of amphotericin B, Posaconazole tablets were administered along with amphotericin on hospital admission. Posaconazole was administered orally as $300 \mathrm{mg}$ twice daily on day 1 , followed by $300 \mathrm{mg}$ once daily day for four to six weeks. Serum Posaconazole levels were evaluated weekly.

\section{Surgical Techniques}

\section{Endoscopic Nasal and Sinus Debridement}

Extensive nasal debridement of mucosa and bone was done till a good vascular bed was visualized for patients with mild disease. Intraoperatively, discharge and/or crusts were collected for $\mathrm{KOH}$ mount, PAS, GM staining, culture sensitivity and histopathology. Mucosal tissue, as well as involved bone, was sent for frozen section evaluation.

\section{Extended Endoscopic Nasal and Sinus Surgery}

For patients with moderate disease a MED was performed. This facilitated removal of disease from the retro antral or peri antral areas, such as the pterygoid plates, pterygomaxillary fossa and infratemporal fossa.

The lamina papyracea was removed in all patients who presented with orbital involvement on imaging. In patients

Table 1 CT Protocol

128 channel multislice CT scanner; detector configuration: $64 \times 0.625$

Field of view (FOV): $180 \mathrm{~mm}$

Slice thickness: $0.67 \mathrm{mms}$; Slice increment / recon thickness: $0.34 \mathrm{mms}$

Bone algorithm / sharp filters for bone details

Intravenous contrast: $80 \mathrm{ml}$ of Iohexol $350 \mathrm{mg} / \mathrm{I}\left(\right.$ Omnipaque ${ }^{\mathrm{TM}}$; GE Healthcare, ) in a dose of 2.0-3.0 $\mathrm{ml} /$ second with a pressure injector

followed by a saline chaser. Scanning is commenced after a delay of 60-70 s after injection 
Table 2 MRI protocol for patients of Mucormycosis

\begin{tabular}{lll}
\hline Sr. No & Sequence & Imaging plane \\
\hline 1 & 3D FLAIR (fluid attenuated inversion recovery) & Axial, coronal, sagittal \\
2 & EPI DW (diffusion weighted) for brain & Axial \\
3 & SWI ( susceptibility weighted) for brain; optional & Axial \\
4 & STIR (short tau inversion recovery) & Axial, coronal \\
5 & TIW & Axial, coronal \\
6 & Fat suppressed post-contrast TIW & Axial, coronal \\
7 & Non-EPI TSE DW (diffusion weighted) for PNS and orbits; optional & Axial \\
\hline
\end{tabular}

Table 3 Grading system of mucormycosis patients

\begin{tabular}{|c|c|c|}
\hline & Extent & Plan \\
\hline None & Clinical suspicion with negative findings on imaging & $\begin{array}{l}\text { Keep patient on regular follow up in ENT OPD with nasal } \\
\text { endoscopy }\end{array}$ \\
\hline Mild & Involvement of Nasal cavity and Paranasal sinus & Endoscopic nasal Debridement \\
\hline Moderate & $\begin{array}{l}\text { Palate involved (sparing the palatal mucosa) } \\
\text { Premaxillary soft tissue Orbital involvement with no clinical } \\
\text { symptoms or signs of the same }\end{array}$ & $\begin{array}{l}\text { Endoscopic nasal debridement with removal of lamina } \\
\text { papyracea. Denkers approach } \\
\text { Retrobulbar Amphoterecin injection } \\
\text { Sublabial palate mucosa sparing maxillectomy }\end{array}$ \\
\hline Severe & $\begin{array}{l}\text { Palatal Ulcer } \\
\text { Orbital signs -ophthalmoplegia, proptosis, infraorbital swelling, vision } \\
\text { loss-Pterygopalatine Fossa } \\
\text { Infratemporal fossa }\end{array}$ & $\begin{array}{l}\text { Total Maxillectomy } \\
\text { Endoscopic Orbital clearance } \\
\text { Lid sparing Orbital Exenteration }\end{array}$ \\
\hline $\begin{array}{l}\text { Very } \\
\text { severe }\end{array}$ & $\begin{array}{l}\text { Sphenoid bone (Greater / lesser wing) } \\
\text { Clivus } \\
\text { Intracranial extension } \\
\text { (Cavernous sinus, internal carotid artery, meningeal involvement, } \\
\text { brain parenchyma) }\end{array}$ & $\begin{array}{l}\text { Highest tolerable dose of Amphotereci } \\
\text { Maximum possible debridement / drilling the bone } \\
\text { Neurosurgery opinion }\end{array}$ \\
\hline
\end{tabular}

with only inflammatory changes in the orbit (i.e., fat stranding, bulky orbital muscles), retrobulbar amphotericin was injected either transcutaneously by the oculoplastic surgeon or endoscopically. The retrobulbar amphotericin was prepared according to the recommendations of the Ophthalmology Society of India, in which $10 \mathrm{ml}$ sterile water was mixed in $50 \mathrm{mg}$ amphotericin $\mathrm{B}$, and $7 \mathrm{ml}$ of this liquid was then mixed with $3 \mathrm{ml}$ of water, resulting in a final constitution of $3.5 \mathrm{mg}$ in $1 \mathrm{ml}$. Three $\mathrm{ml}$ of this reconstituted solution was injected.

\section{Sublabial Maxillectomy}

In patients with involvement of premaxillary soft tissue and / or involvement of alveolar and palatal bone a sublabial approach was used to remove all the diseased bone and soft tissue sparing the palatal mucosa.

\section{Total Maxillectomy}

Total maxillectomy was performed for patients with involvement of the anterior and lateral walls of the maxilla, subcutaneous tissue of the cheek, alveolar bone and palate. This was performed by either the sub-labial or external approach.

\section{Endoscopic Orbital Clearance}

In patients with intraorbital extension endoscopic orbital debridement was performed. All tissues affected by the disease were removed. The optic nerve was preserved.

\section{Orbital Exenteration}

Lid sparing orbital exenteration was performed when there was involvement of the globe, orbital apex, and intraconal 
tissues with no vision in the affected eye. For this decision, the consent of two surgeons was required.

\section{Postoperative Care}

Patients were asked to use saline nasal washes daily. Nasal endoscopy was performed weekly. Repeated endoscopic nasal debridement was performed in patients showing persistent disease.

The total duration of treatment was individualized for each patient. All patients were advised to continue posaconazole tablets $300 \mathrm{mg}$ daily for 3 months.

Repeat imaging was performed if any new complaints or complications were observed, or in patients not responding to treatment satisfactorily.

\section{Observation and Results}

From January to June 2021, a total of 65 patients with postCOVID19 ROCM were managed at this centre. Fifty one were male and 14 female.

Fifty four were hospitalized and 11 were treated at home for COVID 19. All the hospitalized patients received oxygen during COVID 19 treatment for varied periods of time, ranging from three to 14 days. All the patients received steroids during COVID treatment. Twenty-two patients were known diabetics, 39 patients were detected to have high blood sugar levels during COVID 19 treatment and 4 patients had high blood sugar levels when they presented with ROCM.

According to our grading system, 11 patients had mild disease, 27 patients had moderate disease, 16 patients were severe and 11 patients were very severe.

Twenty patients underwent endoscopic nasal debridement and 14 patients underwent MED. Fourteen patients underwent total maxillectomy, nine of which were performed by an external approach and five using a sublabial approach.

Twenty-four patients were detected to have orbital involvement. Thirteen of these patients underwent lid sparing orbital exenteration, 3 patients underwent endoscopic orbital clearance and retrobulbar amphotericin was administered to 8 patients.

Seven patients died due to mucor-related complications $(10.7 \%)$. All these patients had intracranial involvement.

Thirty-five patients were considered cured, as they have shown good clinical improvement. The remaining patients are still under treatment.

\section{Discussion}

Mucormycosis is an opportunistic fungal infection characterised by infarction and necrosis of host tissues, which results from the invasion of the vasculature by hyphae. ROCM is the most common presentation and is secondary to the inhalation of spores into the nose of a susceptible host. All our patients had ROCM.

The incidence of mucormycosis varies from 0.005 to 1.7 per million population [4].

The mortality rate in mucormycosis infection is $\geq 40 \%$ despite aggressive surgical and polyene antifungal therapy. In patients with hematologic malignancy or HSCT recipients the mortality rates are $65 \%$ and $90 \%$, respectively [8-10]. In our study the mortality rate was $10.7 \%$. All the patients who succumbed to the disease had intracranial involvement. The reduced mortality rate may be due to early diagnosis and intervention of these patients.

In the pre COVID era, the predisposing conditions for mucormycosis were uncontrolled diabetes mellitus, haematological malignancies, severe burns, malnutrition, organ transplantation, long-term chemotherapy or immunosuppressed conditions [11, 12].

The surge of ROCM post-COVID-19 infection was due to inherent neutropenia and lymphopenia, presence of DM/ DKA, available free iron and high use of steroids as a part of treatment [13]. All the patients in this study received steroids. There have been many other theories, such as the use of industrial oxygen, high levels of zinc and hospitalacquired due to infected humidifiers; however, these have not been scientifically proved. In our study, 11 patients (17\%) were neither admitted to a hospital or received oxygen.

Mucormycosis has a reputation of being an acute fatal infection. A high index of suspicion and a low threshold for evaluation is essential for early diagnosis and treatment which reduces morbidity and mortality. Early administration IV amphotericin and extensive surgical debridement was done for all patients as delay in treatment of two to six days increases mortality by more than $50 \%$ [14].

IV amphotericin (liposomal) is administered in the dose of $5-10 \mathrm{mg}$ per $\mathrm{Kg}$ body weight per day. After initial treatment with IV amphotericin for several weeks along with the achievement of clinical improvement, the amphotericin is usually stepped down to oral posaconazole or isavuconazole. In the step-down therapy, oral posaconazole (delayed-release tablet) is given at a dose of $300 \mathrm{mg}$ twice daily on the first day, followed by $300 \mathrm{mg}$ once daily. There is no evidence for the benefit of a combination of drug therapy in the form of combining amphotericin with an echinocandin at this stage. However, as there was a shortage of amphotericin due to the 
increased demand so all patients were simultaneously administered oral Posaconazole, $300 \mathrm{mg}$ daily. A retrospective study had observed a potential benefit for the above combination therapy in mucormycosis patients with brain involvement, though the conclusion is debatable [15]. Serum concentration of oral posaconazole needs to be monitored after a week of treatment and has to be kept at least above $1 \mathrm{mcg} / \mathrm{ml}$. This was done for all patients.

Non contrast CT scan detects early intra-sinus and perisinus inflammatory changes without accompanying bony wall erosions. CT is also useful in identifying abnormally irregular / necrotic nasal turbinates / septal mucosa. Contrast enhanced CT can diagnose cavernous sinus thrombosis and show extent of necrosis within inflammatory soft tissue and bone. MRI examinations are more accurate than CT studies to in delineating vascular invasion, intracranial / intraorbital extension and in assessment for early perineural spread. A non-contrast T2 weighted MRI with fat saturation or short-TI inversion recovery (STIR) image can raise suspicion for all above findings [16] which are confirmed on post-contrast fat suppressed sequences.

MRI of the sinuses and orbits showed three patterns with largest number of cases showing iso to hypo intense appearance of the fungal pathology on T2W images. The T2 hypointense appearance is due to presence of iron and manganese in the fungal elements [17] Post contrast images showed three patterns, namely, intense enhancement of abnormal soft tissue, heterogeneous, variable enhancement and non-enhancing peri-sinus soft tissue / bone and primary pathology. T2W signal or enhancement patterns, however, are also seen in bacterial infections and are not reliable markers for invasive fungal infection. Careful attention to often subtle extra-sinus extension in the form of stranding in the premaxillary region, retro-antral fat, intra-orbital fat and in the pterygopalatine fossa are more specific pointers suggesting possibility of an invasive fungal infection on imaging, in the appropriate clinical setting. As described in previous literature MRI has proven to be useful in detection of complications like orbital cellulitis, cavernous sinus thrombosis and ICA thrombosis with cerebral infarction [18, 19], A focally non-enhancing nasal turbinate or septal mucosa on post-contrast MRI studies ('black turbinate' sign) is suggestive of under/non-perfused tissue seen as an early sign of invasive fungal involvement (Fig. 1). Other imaging findings include mucosal thickening, opacification of the paranasal sinuses with or without cortical breach of the walls of the sinuses. Peri-sinus extension of the pathology can be identified in the premaxillary fat, retro-antral space and in the pterygopalatine fossa / infratemporal fossa (Fig. 2). Spread to the intraconal or extraconal compartment of the orbit is well seen on axial and coronal images which also identifies presence of pathology in the orbital apex (Fig. 3a), superior and

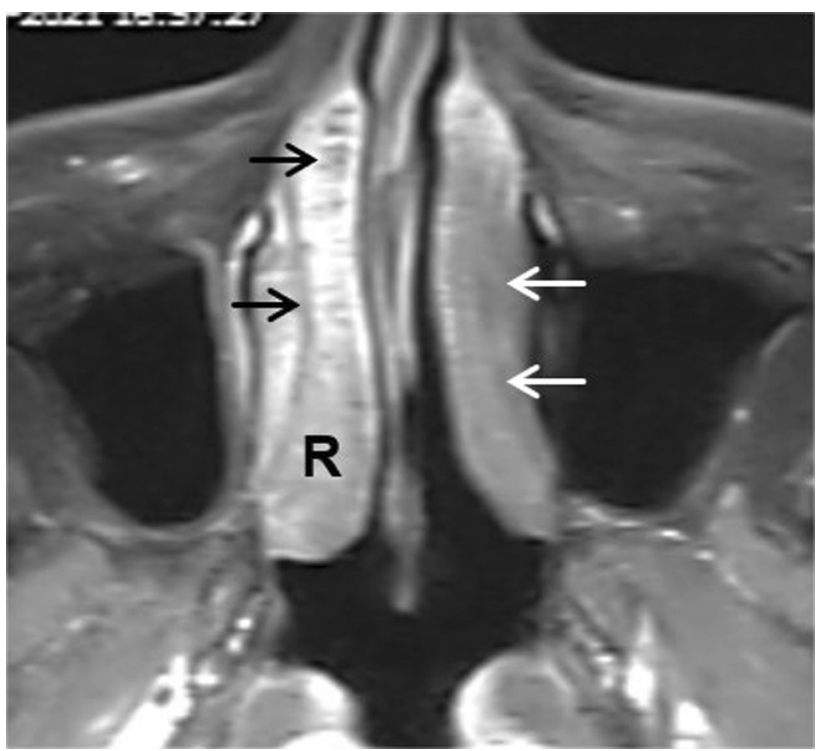

Fig. 1 Axial fat-suppressed post-contrast MR image showing nonenhancing left nasal turbinate (white arrows) depicting the 'black turbinate' sign. Normal turbinate enhancement seen on right side (R). Non-enhancing linear striations on right side (black arrows) are a normal finding

inferior orbital fissures.MRI also demonstrates abnormal signal and enhancement of the optic \& oculomotor nerves, peri-optic nerve sheath (Fig. 3b) and thrombosis of the superior ophthalmic vein / cavernous sinus. Evaluation of the adjacent brain is essential to document spread to the cavernous sinus, internal carotid artery, meninges and cerebral parenchyma. A limited bone high resolution computed tomography (HRCT) is complementary to an MRI examination for identifying marrow oedema in the form of abnormal STIR signal and post-contrast enhancement within the bony structures. Hence, a CT examination after the MRI was also performed wherever deemed necessary to evaluate the bony sinus walls, alveolar ridges, palate and skull base structures.

A review of literature was done for staging of ROCM. Rupa et al developed a 3 tiered staging system [20] and Honovar [21] recently proposed a 4-tiered system. Malhotra $\mathrm{H} \mathrm{S}$ et al. recommended the use of a grid system for the precise localization of disease process and staging [22]. The authors have proposed a grading system correlating the extent with the proposed management plan. A higher grade indicates more extensive surgical intervention.

In the present study, a frozen section of the tissue was sent intraoperatively. This enabled the surgeon to do an extensive surgical debridement immediately and reduced the number of repeated surgical interventions. Literature search proves the role of frozen section in diagnosing mucor infections [23]; however, a comparative study would be ideal to determine the exact sensitivity of frozen section. Intraoperatively tissue was sent for fungal staining 


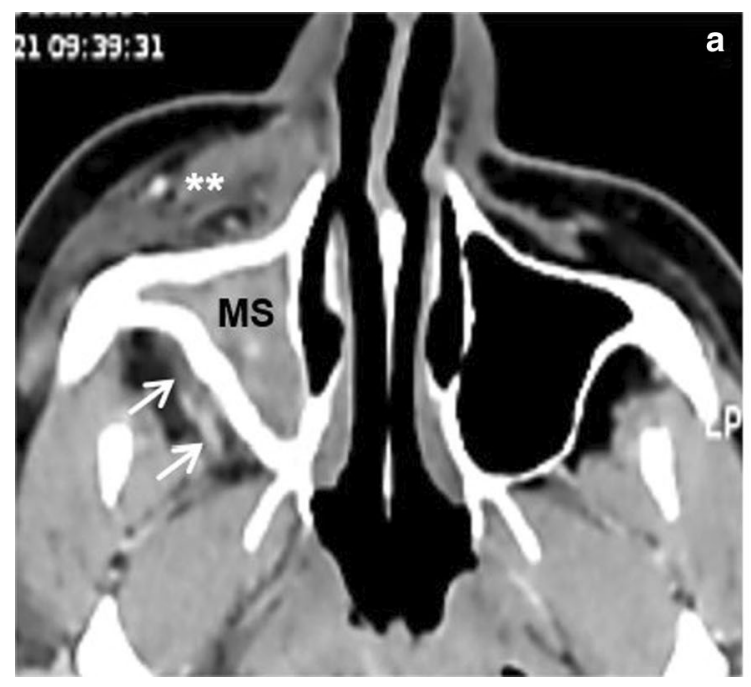

Fig. 2 Non-contrast axial CT image a and axial fat-suppressed postcontrast MR image $\mathbf{b}$ showing peri-sinus extension of the pathology from an opacified right maxillary sinus (MS). Extension can be

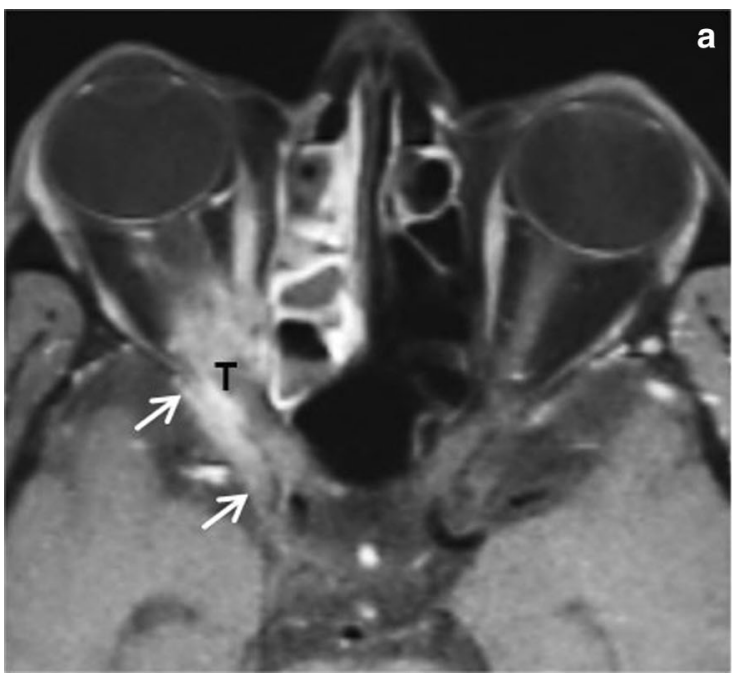

Fig. 3 Axial $\mathbf{a}$ and coronal $\mathbf{b}$ fat-suppressed post-contrast MR images showing enhancing soft tissue (T) at the right orbital apex with intracranial extension (oblique white arrows in a). Retro-orbital enhancing tissue (oblique white arrows in b) is present with

and culture, as well as histopathology to confirm the diagnosis and look for microinvasion.

Aggressive radical surgery has been the mainstay of surgical debridement for mucormycosis. In the past years, advances in technology and expertise have made endoscopic approach the preferred technique. External approaches are reserved for patients with extensive disease e.g., involvement of CNS or orbits [22]. Surgeries in such cases include maxillectomy, orbital exenteration and or craniofacial resection. The aim of surgery is the debridement of necrotic tissue. The extent of debridement is determined by

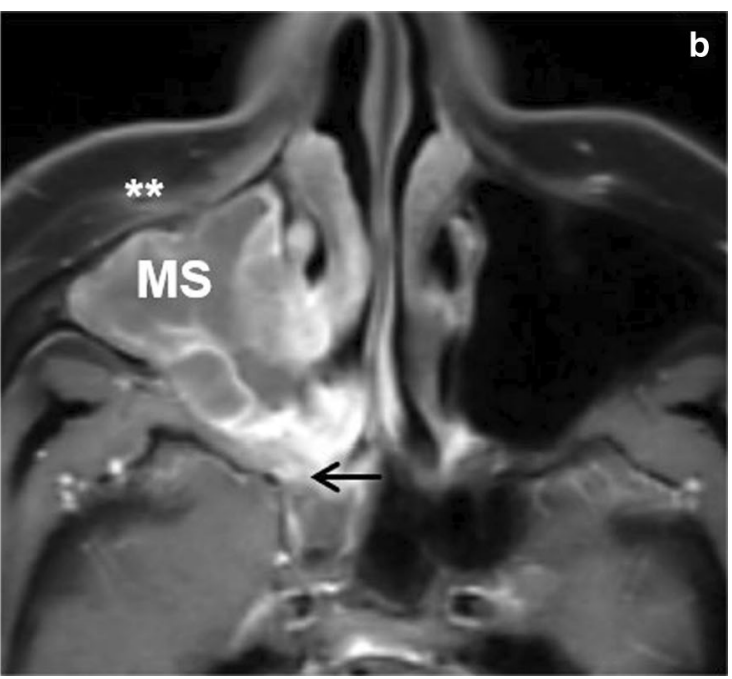

identified in the right sided premaxillary fat (asterisks in $\mathbf{a} \& \mathbf{b}$ ), retroantral space (oblique white arrows in a) and in the pterygopalatine fossa (horizontal black arrow in $\mathbf{b}$ )

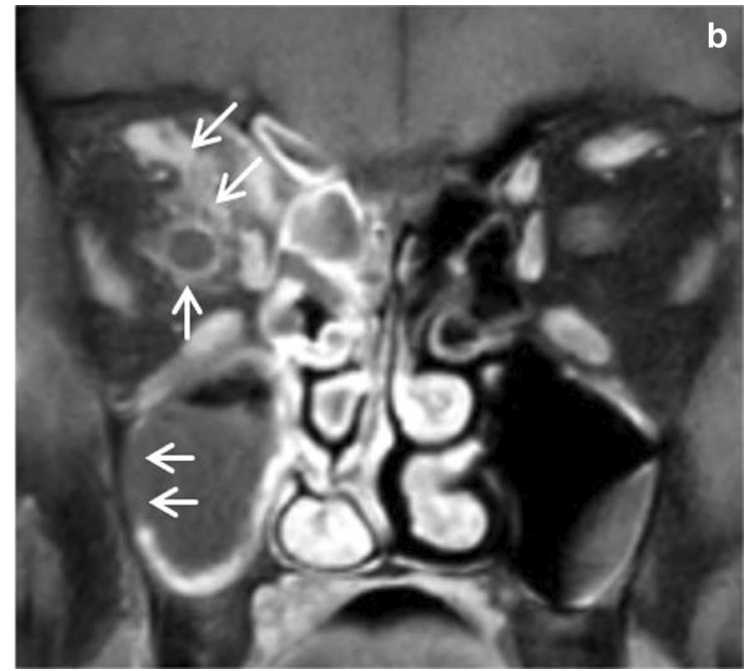

enhancement of the right optic sheath (vertical white arrow in b). Focal necrotic non-enhancing mucosal lining noted in the right maxillary sinus (horizontal white arrows in b)

the ooze of fresh blood from the region of involvement. The enhanced visibility offered by the endoscope allows for better removal of necrotic tissue and preservation of normal tissue [24]. It enhances visibility to anatomical regions like the pterygopalatine and infratemporal fossa. Endoscopic sinus surgery causes less operative morbidity. The MED procedure enables access to the entire maxillary sinus especially the anterior wall. The approach gives a great exposure for clearance of disease from the pterygopalatine and infratemporal fossa. The authors found this 
approach to be very helpful in the management and postoperative surveillance of patients with ROCM.

To summarise:

1) The presentation of mucormycosis patients in the post-COVID scenario is the same as that reported in pre-COVID times.

2) All affected patients presented with high glucose levels at the time of detection of mucormycosis.

3) Grading the disease at presentation assists in the planning of surgical debridement.

4) Immediate debridement with initiation of amphotericin injection due to high suspicion of mucormycosis is ideal.

5) Intraoperative frozen section assists in rapid diagnosis and subsequent extensive surgical debridement.

6) Posaconazole, as an adjuvant drug, is beneficial,

7) Various possible modalities of debridement and disease clearance may be utilised and the plan should be customized for each patient.

\section{Conclusion}

There has been a significant escalation of cases of rhinoorbital-cerebral mucormycosis infection in post-COVID patients. All patients with mucormycosis presented with high blood sugar levels. Early diagnosis, aggressive surgical debridement and antifungal drug therapy is the key to improve survival. Procedures such as endoscopic orbital clearance, sublabial maxillectomy, and Denker's approach may facilitate access and surgical debridement. Posaconazole has emerged as an effective alternate/adjunct antifungal drug in the management of these patients; however, more randomised trials are required.

The new staging system proposed in this study assists in the proper counselling of patients and planning the approach and extent of surgical debridement.

With a fear of a third wave involving more mutations of the virus, this experience of mucormycois definitely provides insight regarding its causes in a tertiary care Indian centre managing both COVID infections and now mucormycosis. Additionalsimilar reports are requiredfor a better understanding of the outcomes of this invasive fungal infection.

Acknowledgements We acknowledge the whole hearted support of all the members of hospital mucor board in patient management. Department of medicine, ophthalmology, microbiology, pathology and Radiology

\section{Declarations}

Conflict of interest The authors declared that there is no conflict of interests.

Informed Consent Obtained for all study patients.

\section{References}

1. Binder U, Maurer E, Lass-Flörl C (2014) Mucormycosis-from the pathogens to the disease. Clin Microbiol Infect 20(Suppl 6):60-66

2. Reid G, Lynch JP 3rd, Fishbein MC, Clark NM (2020) Mucormycosis. Semin Respir Crit Care Med 41(1):99-114

3. Hernández JL, Buckley CJ. Mucormycosis. [Updated 2020 Jun 26]. In: Stat Pearls [Internet]. Treasure Island (FL): Stat Pearls Publishing; 2021 Jan-. Available from: https://www.nc bi.nlm.nih.gov/books/NBK544364/

4. Chamilos G, Lewis RE, Kontoyiannis DP (2008) Delaying amphotericin B-based frontline therapy significantly increases mortality among patients with hematologic malignancy who have zygomycosis. Clin Infect Dis 47:503-509

5. Jeong W, Keighley C, Wolfe R (2019) The epidemiology and clinical manifestations of mucormycosis: a systematic review and meta-analysis of case reports. Clin Microbiol Infect 25:26-34

6. Chander J, Kaur M, Singla N (2018) Mucormycosis: battle with the deadly enemy over a five-year period in India. J Fungi 4(2):46

7. Prakash H, Chakrabarti A (2019) Global epidemiology of mucormycosis. J Fungi 5:26

8. Roden MM, Zaoutis TE, Buchanan WL et al (2005) Epidemiology and outcome of zygomycosis: a review of 929 reported cases. Clin Infect Dis 41:634-653

9. Spellberg B, Edwards J Jr, Ibrahim A (2005) Novel perspectives on mucormycosis: pathophysiology, presentation, and management. Clin Microbiol Rev 18:556-569

10. Spellberg B, Walsh TJ, Kontoyiannis DP, Edwards J Jr, Ibrahim AS (2009) Recent advances in the management of mucormycosis: from bench to bedside. Clin Infect Dis 48(12):1743-1751

11. Papadogeorgakis N, Parara E, Petsinis V, Vourlakou C (2010) A case of successfully treated rhinocerebral mucormycosis: dental implications. Int J Dent. https://doi.org/10.1155/2010/273127

12. Sugar AM (1992) Mucormycosis. Clin Infect Dis 14:S126-S129

13. Singh AK, Singh R, Joshi SR, Misra A (2021) Mucormycosis in COVID-19: A systematic review of cases reported worldwide and in India. Diabetes MetabSyndr 15(4):102146

14. Werthman-Ehrenreich A (2021) Mucormycosis with orbital compartment syndrome in a patient with COVID-19. Am J Emerg Med. https://doi.org/10.1016/j.ajem.2020.09.032

15. Reed C, Bryant R, Ibrahim AS, Edwards J, Filler SG, Goldberg R, Spellberg B (2008) Combination polyene-caspofungin treatment of rhino-orbital-cerebral mucormycosis. Clin Infect Dis 47(3):364-371

16. Ajith Kumar AK; Vikas Gupta, Stat Pearls (Internet)

17. Terk MR, Underwood DJ, Zee CS, Colletti PM (1992) MR imaging in rhino cerebral and intracranial mucormycosis with CT and pathologic correlation. Magn Reson Imaging 10:81-87

18. McDevitt GR, Brantley MJ, Cawthon MA (1989) Rhinocerebralmucormycosis: A case report with magnetic resonance imaging findings. Clin Imaging. https://doi.org/10.1016/08 99-7071(89)90065-X

19. Yousem DM, Galetta SL, Gusnard DA, Goldberg HI (1989) MR findings in rhinocerebralmucormycosis. J Comput Assist Tomogr 13:878-882 
20. Rupa V, Maheswaran S, Ebenezer J, Mathews SS (2015) Current therapeutic protocols for chronic granulomatous fungal sinusitis. Rhinology 53(2015):181-186. https://doi.org/10.4193/Rhin 14.183

21. Honavar SG (2021) Code mucor: guidelines for the diagnosis, staging and management of rhino-orbito-cerebral mucormycosis in the setting of COVID-19. Indian $\mathrm{J}$ Ophthalmol 69(6):1361-1365. https://doi.org/10.4103/ijo.IJO_1165_21

22. Malhotra HS, Gupta P, Mehrotra D et al (2021) Covid 19 associated mucormycosis: staging and management recommendations ( Report of a multidisciplinary expert committee). J Oral Biology and Craniofacial Research 1(4):569-580
23. Papagiannopoulos P, Lin DM, Al-Khudari S, Rajan K, Reddy S, Gattuso P, Tajudeen B, Batra PS (2017) Utility of intraoperative frozen sections in surgical decision making for acute invasive fungal rhinosinusitis. Int Forum Allergy Rhinol 7(5):502-507

24. Jiang R-S, Hsu CY (1999) Endoscopic sinus surgery for rhino cerebral mucormycosis. Am J Rhinol 13(2):105-109

Publisher's Note Springer Nature remains neutral with regard to jurisdictional claims in published maps and institutional affiliations. 